\title{
Laparoscopy in Infertility: A Retrospective Analysis of 200 Cases
}

\author{
${ }^{1}$ Charu Pathak, ${ }^{2}$ Rahul Manchanda, ${ }^{3}$ Garima Yadav
}

\section{ABSTRACT}

Study objective: The aim of this study is to analyze the findings in females on whom laparoscopy was performed for the evaluation and management of infertility.

Design: Retrospective study in a Multispecialty Hospital.

Participants: Two hundred patients who underwent diagnostic and Operative Laparoscopy for evaluation and management of infertility in the age group 20-49 years were selected.

Materials and methods: In this retrospective observational study we evaluated 200 patients on whom laparoscopy was performed for evaluation of primary or secondary infertility, and simultaneous management of the cause was done in a gynae-endoscopy unit in Delhi. The study included 120 females of primary Infertility and 80 women with secondary Infertility for 18 months. Laparoscopic findings, patient's age, nature of pathology and their distribution, the most common factor involved in infertility, chromopertubation, and results were analyzed from the data collected from these available case records.

Results: Total 200 women on whom laparoscopy was performed for evaluation of infertility, it was observed that there were 120 cases were of primary infertility, Out of 120, 8 cases had no abnormal finding. A most common finding was endometriosis in 6/120 (57.5\%) cases including endometrioma in $38(31.7 \%)$ cases. Tubal factor 24 cases $(20 \%)$, ovarian factor 14 cases $(11.7 \%)$, bowel and Omental adhesions 28 cases $(23.33 \%)$, PCOD 32 cases $(26.7 \%)$, and myoma 34 cases $(28.33 \%)$, similarly Out of 80 cases of secondary infertility in 11 cases no abnormal finding was there. Strangely, even in cases of secondary infertility, we found endometriosis to be the most common cause in about $53 / 80(66.3 \%)$ cases which is a significant number. This may be the possible reason for adhesions (Bowel, mental and other) also which account to 42 cases $(52.5 \%)$. Tubal factor 13 cases (16.25), ovarian factor 9 cases $(11.25 \%)$, endometriosis 24 cases (30\%), PCOD 4 cases $(5 \%)$, Myoma 17 cases $(21.25 \%)$.In both primary and secondary infertility, filmy dense adhesions were seen distorting the normal anatomy. These findings were not significant according to age groups $20-30$ years and $>30$ years.

\section{${ }^{1}$ ICOG Fellow, ${ }^{2}$ Director, ${ }^{3}$ Fellow \\ 1,3Department of Obstetrics and Gynaecology, Endo Gynae Unit, PSRI Hospital, New Delhi, India \\ ${ }^{2}$ Department of Obstetrics and Gynaecology, Endo Gynae Unit, Manchand's Endoscopic Centre, New Delhi, India}

Corresponding Author: Charu Pathak, ICOG Fellow, Department of Obstetrics and Gynaecology, Endo Gynae Unit, PSRI Hospital, New Delhi, India, e-mail: drrahulmanchanda@rediffmail.com
Conclusion: In this study, endometriosis was found to be the most significant factor contributing to both primary and secondary infertility and also giving an explanation to increased finding Bowel/Omental and pelvic adhesions. Sometimes, more than one factor is present in overlapping fashion making management more difficult. Laparoscopy has a key role in delineating and managing such cases. It is the minimally invasive method, reaching new horizons with better technique. Simultaneous chromopertubation can be done for evaluation of tubal patency. Now in this era, it is impossible to consider workup for infertility without the aid of laparoscopy.

Clinical significance: This study demonstrates the benefits of laparoscopy in the evaluation of infertility patients and the advantages of the minimally invasive technique.

Statistical analysis: Fischer's exact test for calculation of $p$ value. A $p$ value $<0.05$ shows a statistically significant difference.

Keywords: Adhesions, Endometriosis, Infertility, Laparoscopy, PCOD.

How to cite this article: Pathak C, Manchanda R, Yadav G. Laparoscopy in Infertility: A Retrospective Analysis of 200 Cases. J South Asian Feder Obst Gynae 2018;10(Suppl 2):419-424.

Source of support: Nil

Conflict of interest: None

Date of received: 16 December 2015

Date of acceptance: 4 March 2018

Date of publication: July 2019

\section{INTRODUCTION}

World Health Organization (WHO) defines, infertility as"A disease of the reproductive system defined by the failure to achieve a clinical pregnancy after 12 months or more of regular unprotected sexual intercourse". Incidence of infertility accounts to $10-15 \%$ patients of reproductive age group 1 including both male and female factors which makes it necessary to evaluate the couple rather than an individual.

Before the era of minimally invasive surgeries, it was difficult and almost impossible to evaluate pelvic pathologies. Description of laparoscopy was given by Ott and Kiel in 1901. ${ }^{1}$ The story of laparoscopy has advanced from diagnostic to operative procedures with the advancement in new techniques. Since then new dimensions to the comprehensive and meticulous workup and management of pelvic pathologies have been added.

The advantage of uterine manipulation, visualization of ovaries and tubes for morphology and simultane- 
ous chromopertubation for tubal patency with directed biopsy in suspicious cases of genital tuberculosis or malignancy are potential benefits. With the added advantage of optic magnification, laparoscopy provides an opportunity to pick finest details of disease and not to miss them in early stages especially in cases of endometriosis wherein it is the gold standard method for diagnosis. ${ }^{2}$ Diagnostic laparoscopy has a pivotal role in cases of infertility for direct visualization of abdominal cavity and pelvic organs to explore the deepest of pathologies. With the advent of microsurgical techniques in, a laparoscopic reversal of sterilization has opened a new channel for the management. Disease like endometriosis is better diagnosed and treated simultaneously in the same sitting.

Laparoscopy being minimally invasive technique, associated with less tissue handling and trauma, fewer adhesions, small incision which leads to faster recovery, a short stay at the hospital, cosmetically better scar, less postoperative pain, and cost benefit. Laparoscopy has the edge over laparotomy because of these benefits. Nowadays laparoscopy and hysteroscopy are concomitantly performed invariably in cases of infertility for a thorough evaluation

The main pathological findings encountered during laparoscopy are endometriosis, myoma (multiple including intramural, subserous), adenomyoma, Endometriomas, tubo-ovarian mass, ovarian cysts including polycystic ovaries, Adhesions (bowel and omentum, pelvic, filmy and dense adhesions), hydrosalpinx. Bilateral and unilateral tubal blockage was also seen as a separate entity in the tubal factor without hydrosalpinx. In a few cases, we found perihepatic adhesions suggestive of Fitz-Hugh Curtis syndrome, Koch's abdomen was considered as a differential diagnosis in cases of dense adhesions with the plastered abdomen. Broad uterine fundus with fundal depression was seen in cases of bicornuate uterus, which was simultaneously confirmed by hysteroscopy. In one case of MRKH, uterus was absent with normal tubes and ovaries.

There is a blanket of tests and procedures required to be done as preoperative work up in cases of infertility, including hysterosalpingogram or HSG. Nowadays, combined Laparoscopy and hysteroscopy have seemed to fill the gap of fallacies of HSG. A study by Roma et al. shows that there are $9 \%$ cases of false negative value and $21.85 \%$ cases with false-positive finding, ${ }^{3}$ but its role in initial screening cannot be overruled.

\section{MATERIALS AND METHODS}

The study was conducted in gynae-endoscopy unit of Multispecialty hospital in New Delhi for 18 months. In this retrospective analysis, cases of infertility that underwent laparoscopy were studied, and data was col- lected from available records. Various points considered are-age group of patients, various pathological findings, most common finding in cases of primary and secondary infertility, comparison of findings in both group of patients, to find out number of patients with normal and abnormal findings in age groups $20-\leq 30$ years and $>30$ years in both primary and secondary infertility.

Thorough preoperative workup was done with the selection of patients according to indications; proper counseling and informed consent were also taken into account. Laparoscopy was performed under general anesthesia with standard protocols in the low lithotomy position and $\mathrm{CO}_{2}$ used for pneumoperitoneum with minimum pressure kept at 12-15 $\mathrm{mm} \mathrm{Hg}$.

First, diagnostic laparoscopy was performed with a detailed examination of entire abdominal cavity including upper abdomen (liver, spleen, gall bladder, stomach, omentum, and peritoneum) and lower abdomen including the uterus, both tubes, and ovaries, a pouch of Douglas, ovarian fossa. Simultaneous operative procedures like adhesiolysis, restoration of normal anatomy, excision of ovarian cyst and endometrioma, excision of endometriotic nodules and ablation of superficial spots, myomectomy, tubal reconstruction and Fimbrioplasty, drainage of hydrosalpinx and clipping of the tube, Ovarian drilling was done. These findings according to data were evaluated using Fischer's exact test to calculate $p$ value and $p$ value $<0.05$ was considered significant.

\section{RESULTS}

Out of 200 consecutive patients undergoing Laparoscopy $120(60 \%)$ women were of primary infertility, 80 $(40 \%)$ women had secondary infertility. Various fertility enhancing surgeries were performed simultaneously as indicated.

The age group of patients selected ranging from $20-\leq 30$ years and other groups of $>30$ years for both primary and secondary Infertility cases. Out of 120 patients of primary infertility, $90(75 \%)$ women aged $\leq 30$ years while 30 ( $25 \%$ ) were $>30$ years. Similarly, in secondary infertility group the age of patients range from 20 to 49 years wherein 39 out of $80(48.7 \%)$ women aged $\leq 30$ years and $41(51.25 \%)$ women were $>30$ years.

In primary infertility, women $\leq 30$ years of age, 82 cases out of $90(90.8 \%$ ) were found to have some abnormality on laparoscopy whereas, in $>30$ years group other groups of patients of age $>30$ years $69(86.25 \%)$ patients had abnormal findings. This difference is insignificant according to age wise distribution as $p$ value is $>0.05$ (Table 1).

Considering the secondary infertility group, 31/39 (79.4\%) women aged $\leq 30$ years had abnormal laparo- 
scopic findings whereas 38/41 (92.6\%) women aged > 30 years had some or other pathological finding on laparoscopy, which is not significant according to calculated $p$ value which comes out to be 0.111 that is $>0.5$, which means that there is no difference in proportion of normal and abnormal findings in both age groups (Table 2).

On comparing normal and abnormal findings within primary and secondary infertility groups we found that in 109/120 (90.83\%) primary infertility women one or other pathological finding was detected on laparoscopy as against $69 / 80$ (86.25\%) women with secondary infertility. This difference is also not significant $p$ value is $>0.05$ that is 0.359 as shown in Table 3.

In both the groups of primary and secondary infertility, patients undergoing diagnostic and operative laparoscopy multiple pathological findings were found which were operated accordingly in the same sitting. The data of these findings were collected meticulously on the basis of records available and details are shown below in a tabulated form along with their comparing various abnormal findings shown below in (Table 4).

\section{Endometriosis}

This was found in a significant number of cases accounting to overall 122/200 (61\%). This was almost similar in both primary and secondary infertility cases with a minor difference of $8.8 \%$. Various pathological findings in these cases are:

- Superficial endometriosis: In these cases early inflammatory lesions of endometriosis along with variable findings like brown spots, powder burn appearance, flame lesions peritoneal deposits, superficial deposits on uterus, tubes, and ovaries were seen. Total 34/200 $(17 \%)$ patients of infertility were subdivided into 21 $(10.5 \%)$ cases of primary $13(6.5 \%)$ cases of second-

Table 1: Incidence of laparoscopic abnormalities in primary infertility patients of $\leq 30$ years and $>30$ years

\begin{tabular}{llll}
\hline $\begin{array}{l}\text { Laparoscopic } \\
\text { findings }\end{array}$ & $\begin{array}{l}\text { Age } \leq 30 \\
\text { years } \\
(n=90)\end{array}$ & $\begin{array}{l}\text { Age }>30 \\
\text { years } \\
(n=30)\end{array}$ & $p$ value \\
\hline Normal & $08(8.8 \%)$ & $03(10 \%)$ & 1 \\
Abnormal & $82(91.11 \%)$ & $27(90 \%)$ & \\
\hline
\end{tabular}

${ }^{a}$ Fischer exact test

Table 3: Incidence of laparoscopic abnormal findings in primary and secondary infertility patients

\begin{tabular}{llll}
\hline $\begin{array}{l}\text { Laparoscopic } \\
\text { findings }\end{array}$ & $\begin{array}{l}\text { Primary } \\
\text { infertility } \\
(n=120)\end{array}$ & $\begin{array}{l}\text { Secondary } \\
\text { infertility } \\
(n=80)\end{array}$ & $p$ value \\
\hline Normal & $11(9.16 \%)$ & $11(13.75 \%)$ & 0.359 \\
Abnormal & $109(90.83 \%)$ & $69(86.25 \%)$ & \\
\hline
\end{tabular}

${ }^{\mathrm{a}}$ Fischer exact test ary infertility having superficial endometriosis. Ablation of superficial and early lesions was done with bipolar cautery simultaneously during a diagnostic laparoscopy.

- Grade IV endometriosis: Severe endometriosis involving uterosacral, obliteration of pouch of Douglas, deep scarring adhesions between bowel and omentum with the lateral pelvic wall, abdominal wall and uterus tubes and ovaries distorting the normal anatomy were found. There were $21 / 200$ (10.5\%) of infertility, out of which, $10(5 \%)$ are of primary and $9(4.5 \%)$ cases are of secondary infertility.

- Endometrioma/chocolate cyst ovary: Found in $64 / 200$ (32\%) patients of infertility. 38 out of 120 cases of primary Infertility had right, left and bilateral Endometrioma. Similarly, we found 29/80 cases of Endometrioma in secondary infertility group of patients.

\section{Adhesions}

It was also found in most of the patients, mostly Bowel and Omental adhesions in 70/200(35\%) patients of infertility out of which $28(14 \%)$ account to primary infertility and $42(21 \%)$ in secondary infertility cases. Obliteration of pouch of Douglas in 22/200(11\%), dense adhesions $27 / 200(13.5 \%)$, Filmy adhesions 36/200 (18\%), tuboovarian $16 / 200(8 \%)$. Abdominal wall adhesions were found in some cases with the previous history of surgery like cesarean or laparotomy.

Apart from these adhesions in 5 patients of primary infertility in age group $<30$ years we found plastered abdomen with dense adhesions resembling Koch's abdomen with a positive history of pulmonary tubercu-

Table 2: Incidence of laparoscopic abnormalities in secondary infertility patients at $\leq 30$ years and $>30$ years

\begin{tabular}{llll}
\hline $\begin{array}{l}\text { Laparoscopic } \\
\text { findings }\end{array}$ & $\begin{array}{l}\text { Age } \leq 30 \\
\text { years } \\
(n=39)\end{array}$ & $\begin{array}{l}\text { Age }>30 \\
\text { years } \\
(n=41)\end{array}$ & $p$ value \\
\hline Normal & $8(20.5 \%)$ & $3(7.31 \%)$ & 0.111 \\
Abnormal & $31(79.4 \%)$ & $38(92.6 \%)$ & \\
\hline
\end{tabular}

${ }^{\mathrm{a}}$ Fischer exact test

Table 4: Laparoscopic findings in primary and secondary infertility patients

\begin{tabular}{lll}
\hline Laparoscopic findings & $\begin{array}{l}\text { Primary } \\
\text { infertility } \\
(n=120)\end{array}$ & $\begin{array}{l}\text { Secondary } \\
\text { infertility } \\
(n=80)\end{array}$ \\
\hline Endometriosis & $69(57.5 \%)$ & $53(66.3 \%)$ \\
Myoma & $34(28.3 \%)$ & $17(21.25 \%)$ \\
PCOD & $32(26.7 \%)$ & $04(5 \%)$ \\
Adhesions (bowel/omental) & $28(23.3 \%)$ & $42(52.5 \%)$ \\
Pouch of Douglas obliteration & $13(10.85)$ & $09(11.25 \%)$ \\
Tubal factor & $24(20 \%)$ & $13(16.25 \%)$ \\
Ovarian factor & $14(11.7 \%)$ & $09(11.25 \%)$ \\
\hline
\end{tabular}


losis. In 2 cases with a previous cesarean section, bladder densely adhered to the uterus and abdominal wall.

\section{Uterine Abnormalities}

- Uterine fibroids: Most common finding was myoma including intramural, subserous and multiple myomas. Uterine fibroids were more common in primary infertility group about 33/120 (27.5\%) with the percentage of multiple myomas scoring to $36.3 \%$ of all myomas. The uterine cavity was concomitantly inspected hysteroscopically for submucous myomas. Similarly, in our study, we found $17 / 80(21.25 \%)$ cases of myomas in secondary Infertility patients. Location of most myomas is within the body of the uterus but two cases had small cervical fibroids, one in the anterior wall and another lying posteriorly.

- Absent uterus: Only one case with Mayer Rokitansky Kuster Huaser (MRKH syndrome), uterus was absent rest, both tubes and Ovaries were normal.

- Congenital mullerian anomaly: Broad fundus with fundal depression was seen in 5 cases of the bicornuate uterus as confirmed radiologically and hyeroscopically. No other associated anomaly was seen.

\section{Ovarian factor}

Ovarian cysts were found in 19/200 patients (9.5\%) including right side, left the side and bilateral. More commonly on the left side which does not have any significant correlation. Cysts were more in cases of primary infertility of about $14 / 120$ cases (11.7\%) as compared to $5 / 80$ cases in secondary infertility (6.3\%) only. Tubo-ovarian mass was in $15 / 200$ cases $(7.5 \%)$ in total. Again, more in primary Infertility cases 10/120 (8.33\%) than secondary Infertility $4 / 80$ (5\%). Endometrioma was also found, but included in endometriosis and ovarian factor was analyzed excluding endometrioma because of overlapping data.

\section{Tubal Factor}

Most common finding was Hydrosalpinx in about 13/200 $(6.5 \%)$ cases of infertility, mostly bilateral and more in primary infertility that is $12 / 120(10 \%)$ whereas tubal blockage without hydrosalpinx was more common in cases of secondary Infertility that is $7 / 80$ cases $(8.7 \%)$.

- Parafimbrial adhesions were present in 5 cases of infertility and paratubal cysts were in 4 cases overall.

- Left tubal scarring, left tubal constriction and beaded appearance of tube seen in a single case.

\section{Chromopertubation}

Chromopertubation was done in almost all the patients undergoing evaluation and was found positive with free spillage of dye in 76/200 (38\%) patients of infertility, more in primary infertility $51 / 120(42.5 \%)$ close to half of the patients, whereas it was just $25 / 80(31.3 \%)$ in secondary infertility patients.

\section{DISCUSSION}

Laparoscopy is the minimally invasive technique, reaching new horizons in management of Infertility patients. Direct visualization with optical magnification has an added advantage of diagnosing pathologies which cannot be picked up in ultrasound and other radiological tests that's why laparoscopy is now the gold standard method for diagnosis of diseases like endometriosis. ${ }^{2}$

Several findings from this study deserve detailed discussion. The most common anatomic finding in infertility was endometriosis which was significant in both primaries versus secondary Infertility. In primary infertility, endometriosis account to $57.5 \%$ cases while in secondary infertility 66.35 cases, which highlights the prevalence of this entity. Adhesions (including Bowel and Omental adhesions)distorting normal anatomy was the second most common finding in secondary infertility (Figs 1 and 2). Our data are similar to data obtained by different authors citing endometriosis prevalence rates between $9-50 \%,{ }^{4-6}$ in patients with primary infertility. Incidence of Endometriosis is $22.2 \%$ in asymptomatic women and to around $45-82 \%$ in case of severe dysmenorrhea and ranges from $25-50 \%$ in cases of infertility. ${ }^{7}$ About $17-44 \%$ of patients of endometriosis has ovarian endometrioma. ${ }^{8}$

The rates of pelvic adhesions in our studies are higher than those recently reported in Turkish woman by Göçmen et al. ${ }^{9}$ This might be related to the difference in demographic factors as well as the difference in rates of surgical instrumentation between the two patient populations. Adhesions, as found in our study, are an important factor contributing to secondary infertility and

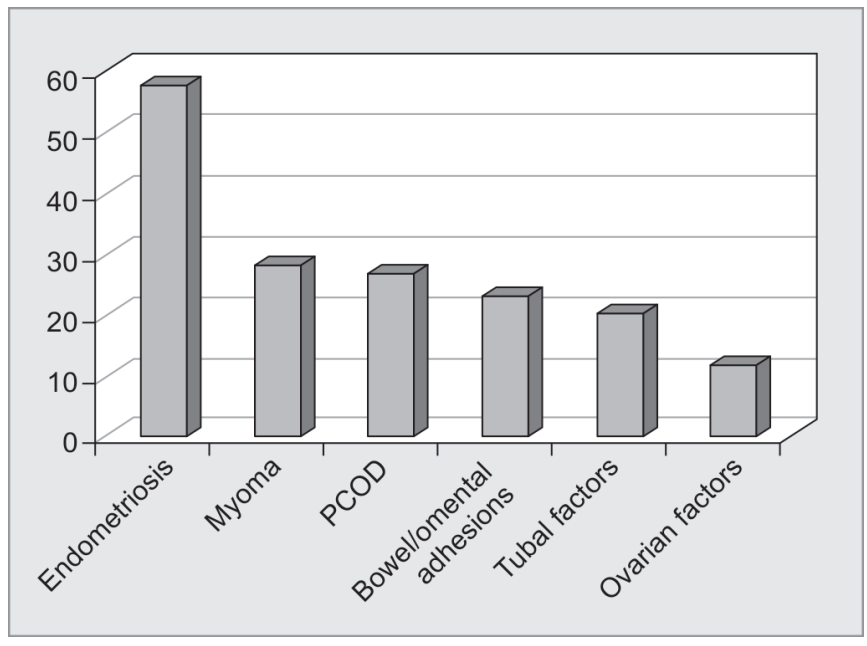

Fig. 1: Common causes of primary infertility as determined by laparoscopy 


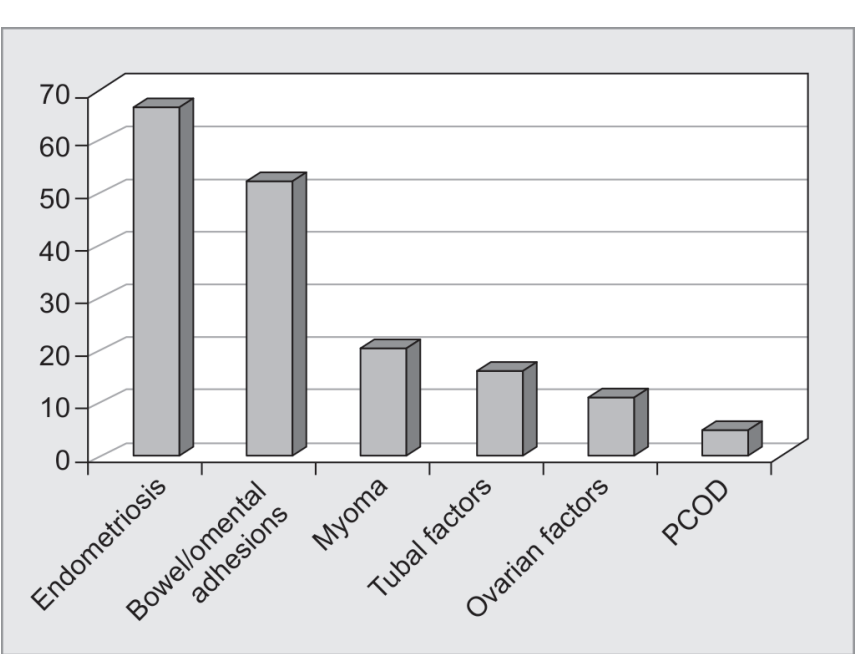

Fig. 2: Common causes of secondary infertility as determined by laparoscopy

are also supported by the literature. ${ }^{10}$ Systematic review of Chocrane database 2009 have shown that adhesiolysis and restoration of Tubal anatomy help improve outcomes in cases of Infertility. ${ }^{11}$ Endometriosis per se, as well as surgical resection of bowel for prior endometriosis, can lead to bowel adhesions and increased risk of secondary infertility.

Age did not appear to be determinant for the presence of anatomic causes of infertility when patients up to 30 years were directly compared with those more than 30 years. A trend towards anatomic abnormality was found but failed to reach statistical significance in woman $>30$ years of age with secondary infertility. However, while anatomic abnormalities may not be distinctly pronounced, physiological changes may account for lower fertility in women with advancing age. Due to the decline in fertility and the increased time to conception occurring with advanced maternal age, women older than 35 years of age should be referred for infertility work-up after 6 months of trying to conceive. ${ }^{12}$ As per our data, PCOD was significantly higher in cases of primary infertility $(26.7 \%)$ than secondary Infertility cases. As per a study conducted by, Hull in a paper published on epidemiology of infertility and polycystic ovarian disease incidence was $21 \%$ of patients of infertility. ${ }^{13}$

Another factor found was, multiple Fibroids present in approximately $5-10 \%$ of the patients presenting with infertility; however, they are found to be the sole identified factor in only $1-2.4 \%$ of the infertile patients. ${ }^{14} \mathrm{~A}$ study conducted by Desai and Patel showed that subserous myoma does not have a significant role in no role in infertility. ${ }^{15-17}$ Intramural fibroids appear to decrease infertility but not proved with evidence yet. As for the management, laparoscopic myomectomy has specific indications considering the location of fibroids and feasibility to operate. In our case, myoma was more in cases of primary infertility as compared to secondary infertility.

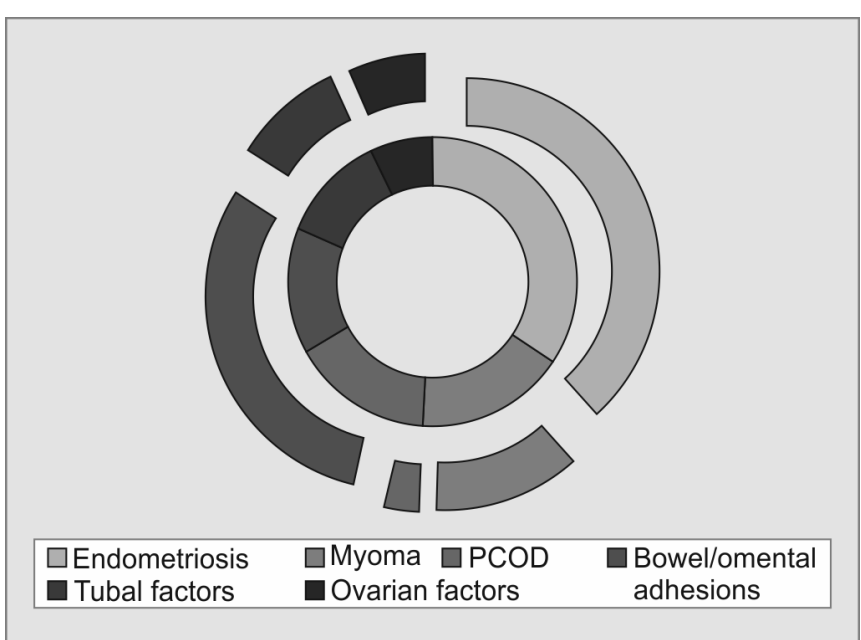

Fig. 3: Split-donut representation for directly comparing the most common causes of primary and secondary infertility: The larger, outer split donut represents the most common causes of primary infertility while the smaller, inner integrated donut represents the most common causes of secondary infertility (as determined by laparoscopy)

This retrospective study shows that more than one anatomic abnormality could be collectively responsible for maternal infertility and these factors overlap each other (Fig. 3). This makes it vital to do a meticulous work up to evaluate and exclude all possible causes of maternal infertility. Also, physiologic and paternal causes can be present which should be considered when laparoscopic studies are unable to delineate an anatomic cause supported by the fact that few cases had no abnormal finding on laparoscopy. Our study has a limitation as of any retrospective study. The small sample size may have precluded the emergence of older age as a statistically significant factor in determining the prevalence of infertility

Multi-center studies with larger sample size, using the consistent, standardized definition for infertility will be needed in the future to determine better the role of various pathologies and anatomic abnormalities contributing to infertility as visualized by laparoscopy. Studies with larger sample size will help better assess the prevalence of anatomic causes of infertility.

The reported overall complication rates of laparoscopy vary from $0.2 \%$ and $10.3 \%$. Complications are more with major laparoscopic procedures as compared with minor procedures, $0.6-18 \%$ and $0.06-7.0 \%$, respectively. ${ }^{18}$ Various complications vary injury to vessel, visceral organs to postoperative ileus. In all 200 cases studied retrospectively, there is only one case reported of postoperative illusion postoperative day 2 . The patient was a case of secondary infertility with grade IV endometriosis and extensive adhesiolysis was done and managed conservatively.

\section{CONCLUSION}

Laparoscopy provides a valuable tool to evaluate the etiology for primary and secondary infertility in females both 
young and with advanced age. Endometriosis was found to be the most common cause responsible for infertility in both primary and secondary infertility cases. There are many pathological factors overlapping with each other which make management challenging in the absence of laparoscopic procedure. This study emphasizes the fact that laparoscopic evaluation is an indispensable tool and is safe in experienced hands.

\section{CLINICAL SIGNIFICANCE}

This study demonstrates the benefits of laparoscopy in the evaluation of infertility patients and the advantages of minimally invasive technique and highlights the fact that there can be more than one anatomic finding overlapping in infertility patients.

\section{REFERENCES}

1. Boivin J, Bunting L, et al. International estimates of infertility prevalence and treatment seeking: Potential need and demand for infertility medical care.Hum Reprod 2007;22:1506-1512.

2. Mishra VV, Gaddagi RA, et al. Prevalance; characteristics and Management of Endometriosis Amongst Infertile Women: A one year Retrospective study. J Clin Diagn Res 2015 Jun;9(6):QC01-QC03. Published online 2015 Jun 1.

3. Dalfó AR, Úbeda B, et al. Diagnostic Value of Hysterosalpingography in the Detection of Intrauterine Abnormalities: A Comparison with Hysteroscopy. AJR 2004;183:1405-1409 0361-803X/04/1835-1405.

4. Cramer DW, Wilson E, et al. The relation of endometriosis to menstrual characteristics, smoking, and exercise. JAMA 1986; 255:1904-1908.

5. Duignan BNM, Jordan JA, et al. One thousand consecutive cases of diagnostic laparoscopy. J Obstet Gynecol Br Commonw 1972;79:1016-1020.
6. Williams TJ, Pratt JR. Endometriosis in 1000 consecutive celiotomies: incidence and management. Am J Obstet Gynecol 1977;129:245-250.

7. Missmer SA, Hankinson SE, et al. Incidence of laparoscopically confirmed endometriosis by demographic, anthropometric, and lifestyle factors. Am J Epidemiol.2004;160:784-796.

8. Kobayashi H, Sumimoto K, et al. OvarianEndometrioma. Risk factors for Ovarian cancer development.Eur J Obstet Gynecol Reprod. Bioi 2008;13892):187-93. Epub 2007 Dec 26.

9. Göçmen A, Atak T. Diagnostic laparoscopy findings in unexplained infertility cases. Clin Exp Obstet Gynecol 2012;39(4):452-453.

10. Honga, Vitz TO, et al. Peritoneal adhesion formation .Chirurg, 2015 Feb; 86(2):175-180.

11. Duff JM, Johson N, et al. Cochrane Database Systematic Rev. 2009 April (2):CD001897.

12. Reproductive Endocrinology and Infertility Committee; Family Physicians Advisory Committee; Maternal-Fetal Medicine Committee; Executive and Council of the Society of Obstetricians, Liu K, Case A.Advanced reproductive age and fertility. J Obstet Gynaecol Can 2011;33(11):1165-1175.

13. Hull MG. Epidemiology of infertility and polycystic ovarian disease: endocrinological and demographic studies. Gynecol Endocrinol 1987;1(3):235-245.

14. Pritts EA.Fibroids and Infertility: a Systematic review of the evidence.Obstet Gynecol Surv 2001;656(8):483-491.

15. Desai P, Patel P et al. Fibroids, infertility and laparoscopic myomectomy. J Gynecol Endosc Surg 2011;2(1):36-42.

16. Dubusson JB, Lecuru $\mathrm{T}$, et al. Myomectomy by Laparoscopy: A preliminary report of 43 cases. Fertil Steril 1991;56: 827-830.

17. Benecke C, Kruger TF, et al. Effect of fibroids on infertility in patients undergoing assisted reproduction: A structured literature review.Gynecol Obstet J 2005;59:225-230

18. Magrina JF. Complications of laparoscopic surgery. Clin Obstet Gynecol 2002;45:469-480. 